\title{
The efficacy of bi-component carboxymethylcellulose-polysaccharide B as a hemostatic and anti-adherent agent at the tibial insertion of the hamstring tendons after reconstruction of the anterior cruciate ligament
}

\author{
D. Costa Astur', F. Caracatto Baras', R. Moukbel Chaim¹, J. J. Krob², \\ G. Gonçalves Arliani ${ }^{1}$, G. Taniguti de Oliveiraa ${ }^{1}$, M. Cohen ${ }^{1}$ \\ 1 Sports Traumatology Divison (CETE) from Departamento de Ortopedia e Traumatologia, \\ Escola Paulista de Medicina (EPM), UNiversidade Federal de São Paulo (UNIFESP), \\ São Paulo, SP, Brazil \\ 2 University of Illinois, Chicago, United States
}

\section{CORRESPONDING AUTHOR:}

\section{Felipe Baras}

Departamento de Ortopedia

e Traumatologia, Escola

Paulista de Medicina (EPM),

Universidade Federal de São

Paulo (UNIFESP), São Paulo,

SP, Brazil

E-mail: fcbaras@gmail.com

DOI:

10.32098/mltj.01.2019.02

\begin{abstract}
SUMMARY
Objective. The objective of this study is to evaluate and compare hemostatic and anti-adherent efficacy of the bi-component carboxymethylcellulose-polysaccharide B to the standard of electocauterization at the gracilis and semitendinosis harvest site in patients undergoing anterior cruciate ligament (ACL) reconstruction. Methods. In this randomized control trial, 32 patients undergoing ACL reconstruction were randomly divided into two groups. In group 1 (control), hemostasis was achieved via electrocoagulation at the gracilis and semitendinosis harvest site, and in group 2 (intervention) the bi-component was used to control bleeding at the same site. Results. There was no statistical difference between the groups in the pain assessments postoperatively, and the patients in group 2 had lower incidence of hematoma than the patients in group 1 on the first day postoperatively. There was a statistically significant difference between the groups in terms of postoperative range of motion $(\mathrm{ROM})$ during the study period $(\mathrm{p}=0.022)$. Patients in group 1 gained 17.8 degrees, patients in group 2 increased ROM by 34 degrees. No statistically significant difference was found in the incidence of hypoesthesia between the groups $(\mathrm{p}=0.870)$. Conclusion. Bi-component carboxymethylcellulose-polysaccharide B showed better outcomes in terms of hematoma prevention and postoperative ROM than standard electrocoagulation in the immediate postoperative period for patients undergoing ACL reconstruction. Surgeons should consider using this compound to achieve hemostasis at the gracilis and semitendinosis harvest site during ACL reconstruction.
\end{abstract}

KEYWORDS

carboxymethyl cellulose sodium; anterior cruciate ligament; knee/surgery; tendon

\section{INTRODUCTION}

Despite being a highly popular graft choice, the comorbidities resulting from the harvest of gracilis and semitendinosus muscle tendons to the anterior cruciate ligament (ACL) reconstruction are a substantial source of patient morbidity and disturb rehabilitation in the early postoperative period $(1,2)$.
Several complications are associated with hamstring tendon graft harvest, including hypoesthesia to the leg due to iatrogenic injury of the infrapatellar branch of saphenous nerve $(3,4)$, and subcutaneous residual hematoma in the place of the incision, causing pain, inflammation, wound dehiscence and increasing patient's recovery and rehabilitation time (5). 
The specific ratio bi-component carboxymethyl cellulose-polysaccharide $\mathrm{B}$ is a natural product that acts as a hemostatic and as an anti-adherent barrier $(6,7)$. The carboxymethyl cellulose component is a product derived from cellulose with low molecular weight and is highly water soluble (8). Its mechanism of action is two-fold. First, it decreases the formation of subcutaneous adherence and fibrosis through the creation of a film that mechanically insulates underlying tissues, thus acting as healing reorganization agent. Second, it forms crosslinks with the second component, polysaccharide $\mathrm{B}$, bestowing the capability to act as a natural hemostatic scaffold. Thus, it controls local bleeding through improving chemical integration between the hydrophilic particles of the polysaccharide and blood cells. The carboxymethyl cellulose component serves as a short-acting coagulation agent, as it is rapidly metabolized by human enzymes. Conversely, the polysaccharide- $\mathrm{B}$ component has a prolonged acting time, improving local hemostasis for approximately forty days (9-11).

The current standard of hemostasis via electrocauterization is not without consequence. The process causes carbonization of the tissues and occasional residual bleeding, leading to postoperative symptomatology. Therefore, the objective of this study is to evaluate and compare hemostatic and anti-adherent efficacy of the bi-component carboxymethylcellulose-polysaccharide B to the standard of electocauterization at the gracilis and semitendinosis harvest site in patients undergoing anterior cruciate ligament (ACL) reconstruction. The hypothesis of this study is that applying bi-component carboxymethyl cellulose-polysaccharide B at the site of hamstring tendon harvest as a hemostatic and anti-adherent agent will result in lower incidence of postoperative hematoma and hypoesthesia, and higher objective function measurements than electrocautery.

\section{METHODS AND MATERIALS}

A clinical randomized control trial was conducted with 32 patients between the ages of 18 and 45 years old undergoing anatomic ACL reconstruction with hamstring autograft. The inclusion criteria were: isolated ACL injury or with the injury of the medial meniscus diagnosed on physical examination, and confirmed by magnetic resonance imaging (MRI); graft thickness equal to or larger than $8 \mathrm{~mm}$, after measurement and adequate preparation. The exclusion criteria were: thickness less than $8 \mathrm{~mm}$; inability of using the semitendinosis and gracilis tendons as a graft for any reason; other associated soft tissue lesions; local infections; or patients with autoimmune and systemic diseases. In addition, patients who chose not to continue the study through full follow-up or refused to take part of it were excluded from analysis.
The morbidity at the incision site was evaluated following hamstring graft harvest. Patients were randomly divided into two groups through sealed envelopes; with group 1 (control) receiving electrocoagulation around the incision and in the deep planes to control bleeding, and group 2 (intervention) was treated with the application of bi-component carboxymethyl cellulose-polysaccharide B (Adhesion ${ }^{\circledR}$, $\mathrm{DMC}$ ) to achieve hemostasis at the harvest site.

\section{Surgical technique}

After inflating the pneumatic tourniquet, the procedure began with the harvest of the semitendinous and gracilis tendons through a $3 \mathrm{~cm}$ vertical incision, approximately $2 \mathrm{~cm}$ medial and distal to the anterior tibial tuberosity. Harvest of each tendon and its extraction were performed using a closed-type tenotomy technique. The arthroscopic ACL reconstruction then followed using previously described anatomical reconstruction techniques (12). Tibial fixation was achieved with an interference screw, and femoral fixation with a cortical button construct.

\section{Application of bi-component carboxymethyl cellulose-polysaccharide B}

The procedure ended with the release of the pneumatic tourniquet. Hemostatis of the surgical wound was achieved with the aid of electrocautery in patients in group 1, and with the local application of bi-component carboxymethyl cellulose-polysaccharide B in group 2 patients. One gram of the powdered compound was applied to the wound until fully covered. Subcutaneous and skin closure followed (figure 1).

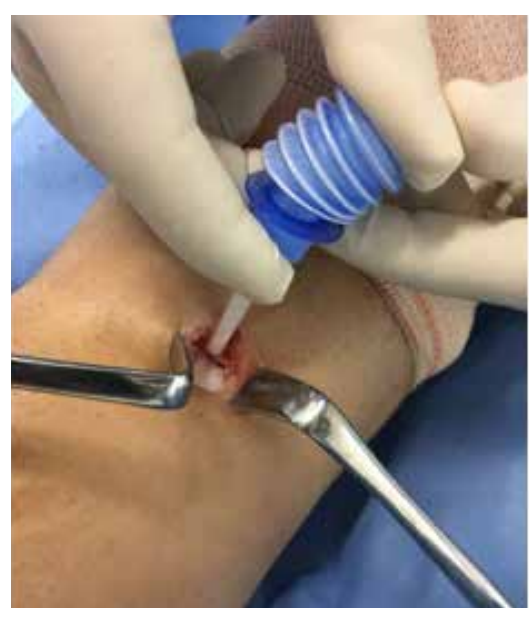

Figure 1 - Applying the hemostatic/anti-adherent component around the wound. 


\section{Rehabilitation and follow-up}

All patients were discharged the day after surgery. Upon discharge, subjective pain scores were recorded, and all patients were examined for hematoma at the harvest incision site and range of motion. All patients were given the same medical regimen after discharge from hospital: diclofenac sodium $50 \mathrm{mg} \mathrm{q} 12 \mathrm{~h}$ for five days, acetaminophen $325 \mathrm{mg} \mathrm{q} 6 \mathrm{~h}$ for five days and cyclobenzaprine $10 \mathrm{mg}$ $\mathrm{q} 12 \mathrm{~h}$ for five days. During the first seven postoperative days, patients were asked about pain, swelling and ROW. Repeated neurological exams were completed to evaluate hypoesthesia due to damage to the infra-patellar branch of the saphenous nerve.

The clinical parameters were evaluated using the visual analogue scale (VAS) to measure the patient's pain (where zero means "no pain" and 10 "worst imaginable pain"). The local hematoma was evaluated according to the following criteria: presence of bulging (4), large volume bulging without significant local pain (3), small volume bulging (2), and without bulging (1).

The ROM examination was performed with the patient in the supine position and with a goniometer fixed to the lateral epicondyle of the femur. The proximal arm of the instrument was positioned over the midline of the femur using the greater trochanter as a reference. The distal arm was oriented parallel to the midline of the fibula using the lateral malleolus and the fibular head as references.

The neurological examination assessed the loss of sensitivity to light touch over the anteromedial aspect of the tibia, secondary to injury to the infrapatellar branch of the saphenous nerve. Hypoesthesia is defined by the sensation of light touch comparing both limbs simultaneously. This area is then demarcated with a pen under the skin.
All patients underwent the same rehabilitation program. Immediately after the surgery, patients began to perform isometric contractions of the quadriceps and hamstring muscles. They were encouraged to initiate partial weight bearing with the aid of crutches. Knee immobilizers were not prescribed, with the intention of beginning strengthening as early as possible. At 14 days postoperatively, full knee extension was expected. Physical therapy continued for at least six months until return to sport.

\section{Statistical analysis}

Statistical analysis was performed using a parametric ANOVA test to compare means obtained at the different analysed moments. To measure the relationship between groups for hypoesthesia, the Chi-square test was also used. It was used a significance level of $0.05(5 \%)$ and $95 \%$ of confidence interval.

\section{RESULTS}

32 patients were included in the study: 16 patients assigned to group 1 , and 16 patients assigned to group 2 . However, one patient in group 2 was excluded because he did not complete follow-up after surgery. On average, the patients were 25 years old (range 19-45 years) at the time of surgery. 30 patients were male, only 2 were female. There was no statistical difference between the groups in terms of pain scores across the evaluation period $(\mathrm{p}>0.05)$. However, it should be mentioned that the scores obtained for the patients in group 2 were always lower than those in group 1 (table I). The average reduction in pain score between the first and seventh day of evaluation was 2.31 points in group 1 and 2.13 points in group $2(\mathrm{p}>0.05)$.

Table I - Comparison between pain scores on VSA in the immediate postoperative period.

\begin{tabular}{|c|c|c|c|c|c|c|c|c|c|}
\hline Pain & & average & median & SD & $\min$ & $\max$ & $\mathbf{n}$ & CI & p-value \\
\hline \multirow{2}{*}{ day 1} & control (G1) & 5.31 & 7 & 3.03 & 0 & 9 & 16 & 1.48 & \multirow{2}{*}{0.385} \\
\hline & intervention (G2) & 4.53 & 5 & 1.64 & 2 & 8 & 15 & 0.83 & \\
\hline day 3 & intervention (G2) & 3.73 & 3 & 1.62 & 1 & 7 & 15 & 0.82 & 0.083 \\
\hline day 5 & control (G1) & 3.75 & 3.5 & 1.73 & 1 & 7 & 16 & 0.85 & 0.137 \\
\hline \multirow{2}{*}{ day 7} & control (G1) & 3.00 & 3 & 1.59 & 0 & 6 & 16 & 0.78 & \multirow{2}{*}{0.316} \\
\hline & intervention (G2) & 2.40 & 3 & 1.68 & 0 & 5 & 15 & 0.85 & \\
\hline \multirow{2}{*}{ gain } & control (G1) & -2.31 & -2.5 & 2.85 & -7 & 4 & 16 & 1.39 & \multirow{2}{*}{0.822} \\
\hline & intervention (G2) & -2.13 & -2 & 1.13 & -4 & -1 & 15 & 0.57 & \\
\hline
\end{tabular}

SD, standard deviation; $\mathrm{CI}=95 \%$ confidence interval. 
Patients in group 2 had a lower incidence of hematomas than patients in group 1 on the first day $(\mathrm{p}=0.008)$. However, there was no difference in incidence between the groups at 7 days postoperatively (table II). If present, the hematomas in group 2 were smaller than group 1 on the first day $(\mathrm{p}=0.008)$. There was a reduction of 1.5 points on average in terms of the hematoma classification scale described above, after seven days for patients in group 1 and 0.87 points on group 2, resulting in a similar score after seven days $(\mathrm{p}>0.05)$.

The average ROM in group 1 after seven days was 73.1 degrees, and 93.7 in group $2(\mathrm{p}=0.013)$. The gain in the range of motion over the first 7 days was significantly greater in group 2 (34 degrees increase) than group 1 (17.8 degrees) $(\mathrm{p}=0.022)$ (table III).

No statistically significant difference was found between the groups for the occurrence of hypoesthesia $(\mathrm{p}=0.870) .43 .8 \%$ of patients in group 1 and $46.7 \%$ of patients in group 2 developed sensation deficits after seven days postoperatively.

In addition to the evaluated outcomes, other inherent complications of the harvest site were recorded. Three patients in group 2 presented signs of hyperemia, heat and flushing. One patient presented with serosanguinous wound drainage and was treated as localized, superficial infection with oral antibiotic therapy for 14 days with complete regression of symptoms. There were no other reported postoperative complications.

\section{DISCUSSION}

The present study partially supported the hypothesis that applying bi-component carboxymethyl cellulose-polysaccharide $\mathrm{B}$ at the site of hamstring tendon harvest as a hemostatic and anti-adherent agent will result in lower incidence of postoperative hematoma and higher objective function measurements than electrocautery, although the incidence of hypoesthesia was not affected. The combined action of a hemostatic with an anti-adhesion barrier benefited patients in their early days of postoperative recovery from ACL reconstruction with hamstring autograft. These patients had a non-significant lower fluid volume at the harvest site, as well as significantly greater knee flexion capacity and gain in ROM over the first 7 postoperative days. The results of this study support the efficacy of the hemostatic capacity of the bi-component to prevent the spread of bleeding as well as increase its residual absorption in the immediate postoperative period.

Electrocautery is one of the most common methods of achieving hemostasis (1). However, by performing electrocautery of the tissues, surgeons cause carbonization, that is, we controlled the bleeding by damaging the tissue around it. Finding a less damaging alternative means of hemostasis may minimize or eliminate these potential risks to patient morbidity. Immediate postoperative pain, swell-

Table II - Comparison between groups for incidence of harvest site hematoma in the immediate postoperative period.

\begin{tabular}{|c|c|c|c|c|c|c|c|c|c|}
\hline \multicolumn{2}{|c|}{ Hematoma } & \multirow{2}{*}{$\begin{array}{c}\text { average } \\
3.19 \\
\end{array}$} & \multirow{2}{*}{$\frac{\text { median }}{3}$} & \multirow{2}{*}{$\frac{\text { SD }}{0.75}$} & \multirow{2}{*}{$\frac{\min }{2}$} & \multirow{2}{*}{$\frac{\max }{4}$} & \multirow{2}{*}{$\frac{\mathbf{N}}{16}$} & \multirow{2}{*}{$\begin{array}{c}\text { CI } \\
0.37 \\
\end{array}$} & \multirow{2}{*}{$\frac{p \text {-value }}{0.008}$} \\
\hline $\operatorname{dav} 1$ & control & & & & & & & & \\
\hline day 1 & intervention & 2.47 & 2 & 0.64 & 2 & 4 & 15 & 0.32 & \\
\hline day 7 & intervention & 1.60 & 1 & 0.74 & 1 & 3 & 15 & 0.37 & \\
\hline gain & control & -1.50 & -2 & 0.97 & -3 & 0 & 16 & 0.47 & 0.05 \\
\hline
\end{tabular}

SD, standard deviation; $\mathrm{CI}=95 \%$ confidence interval.

Table III - Range of motion on physical exam in immediate postoperative period.

\begin{tabular}{llccccccccc}
\hline ADM & & average & median & SD & min & $\max$ & N & CI & p-value \\
\hline \multirow{2}{*}{ day 1 } & control & 55.3 & 47.5 & 33.8 & 0 & 115 & 16 & 16.6 \\
\cline { 2 - 9 } & intervention & 59.7 & 60 & 21.2 & 25 & 90 & 15 & 10.7 & 0.673 \\
\multirow{2}{*}{ day 7 } & control & 73.1 & 77.5 & 28.1 & 15 & 110 & 16 & 13.8 \\
\hline \multirow{2}{*}{ gain } & intervention & 93.7 & 90 & 10.4 & 70 & 110 & 15 & 5.3 & $\mathbf{0 . 0 1 3}$ \\
& control & 17.8 & 15 & 17.0 & -5 & 65 & 16 & 8.3 \\
\hline
\end{tabular}


ing, hematoma formation and, consequently, decreased ability to perform flexion extension movement may be related to this practice $(5,9)$.

Successful ACL reconstruction surgeries tend to be very painful immediately post-operatively, and thus making optimal rehabilitation difficult (13). Much of this pain is thought to be generated from extraarticular procedures, such as graft harvest used to reconstruct the ligament. Pain and hematoma at the site of the hamstring harvest are frequent, and inhibit flexion and extension movement of the knee. For this reason, these outcomes were chosen for evaluation in the present study.

The application of bi-component carboxymethyl cellulose-polysaccharide B directly to the harvest site decreased the immediate incidence of hematomas as hypothesized, supporting the early benefits of this modality of hemostatic action. The improvement in the gain of movement over the first 7 postoperative days also suggests that the secondary insulating film formed by the bi-component carboxymethyl cellulose-polysaccharide B decreased of the formation of fibroses and adhesions. To the authors' knowledge this is a novel result in the literature.

However, when comparing the groups for the pain outcome, there was no significant difference $(\mathrm{p}>0.05)$. Considering that pain symptoms at the site of graft removal is secondary to the local distension produced by the hematoma, we believe that the benefits of hemostasis may be more apparent at a later postoperative date, due to the decrease in the local fluid collection.

Difficulty to differentiate intra-articular pain from the graft harvest site pain, this may be the most plausible explanation for non-significant pain reduction results. Kong et al. (9) evaluated the efficacy of isolated carboxymethyl cellulose in 15 patients undergoing bilateral total knee arthroplasty. This study also reported no differences in pain and joint stiffness between groups.

The authors agree with the literature that the paresthesia caused by the inferior saphenous nerve iatrogenic injury is related to the operative technique, the size and orientation of the incision, and the care with the dissection of the layers than as a result of localized compressive swelling $(14,15)$. Nonetheless, this study aimed to compare the incidence of

\section{REFERENCES}

1. Lopes TJA, Simic M, Pappas E. Epidemiology of anterior cruciate anterior ligament reconstruction in Brazil's public health system. Rev Bras Med Esporte 2016; 22(4):297-301.

2. De Lima LC, Arantes G, Oliveira RV et al. Anatomical reference point for harvesting a flexor graft during arthroscopic this neurological alteration when using different methods of achieving hemostasis, and found no difference between modalities. The percentage of patients who presented with hypoesthesia of the lateral leg was $43.8 \%$ in the patients in group 1 and $46.7 \%$ in the patients in group 2, values similar to those found in the literature (39-55\% of the cases) (14). The study provides evidence that achieving hemostatsis by direct application of bi-component carboxymethyl cellulose-polysaccharide B may be an efficacious alternative for the control of common, immediate postoperative graft harvest site symptoms in patients undergoing ACL reconstruction. The bi-component has been shown to be safe and effective. Even so, the authors acknowledge some limitations of the present study including the number of patients evaluated. Future studies should examine the effects of this compound in a large randomized control study or cohort. Additionally, the present study examines only the immediate postoperative outcomes. Future studies should examine more distant postoperative outcomes with longer follow-up. Moreover, different bi-component carboxymethyl cellulose-polysaccharide B formulations are in production. The present study only evaluated one commercial formula, since this is the only one available in the market, and future studies may examine differences between these compounds.

\section{CONCLUSION}

The use of the bi-component carboxymethyl cellulose-polysaccharide B presented better results in hematoma control and gain of range of motion in the immediate postoperative period following ACL reconstruction with hamstring autograft. It did not change the patient's pain outcomes in the first few postoperative days, nor did it influence the incidence of paresthesia due to injury to the infra-patellar branch of the saphenous nerve $(p>0.05)$. Surgeons should consider its use in the appropriate patient population to achieve hemostasis and reduce patient morbidity.

\section{Conflict of interests}

The authors declare that there is no conflict of interest.

reconstruction of the anterior cruciate ligament. Rev Bras Ortop 2015; 50(2):164-167.

3. Padua VB, Silva SC, Canuto SM et al. Saphenous nerve injury during harvesting of one or two hamstring tendons for the anterior cruciate ligament reconstruction. Rev Bras Ortop 2015; 50(5):546-549. 
4. Inderhaug E, Strand T, Solheim E. The impact of sensory deficits after harvesting hamstings autograft for ACL reconstruction. Knee Surg Sports Traumatol Arthrosc 2015; 23(4):1060-1064.

5. Pinczewski L, Lyman J, Salmon L, Russell V, Roe J, Linklate J. 10-Year Comparison of anterior cruciate ligament reconstructions with hamstring tendon and patellar tendon autograft. Am J Sports Med 2016; 35(4):564-574.

6. Munsterman AS, Kottwitz JJ, Reid HR. Meta-Analysis of the effects of adhesion barriers on the adhesion formation in the horse. Veterinary surgery 2016; 45(5):587-595.

7. diZerega GS, Cortese S, Rodgers KE, et al. A modern biomaterial for adhesion prevention. Journal of biomedical materials research part b: applied biomaterials 2017; 81:239-250.

8. Basilio PC. Intestinal bowel obstruction: use of bio-resorbable membrane (sodium hyaluronate + carboxymethyl cellulose) seprafilm ${ }^{\circledR}$ in the prophylaxis of complications in abdominopelvic reoperations. Rev Bras Coloproct 2003; 23(3):168-71.

9. Kong CG, In Y, Cho HM, Suhl KH. The effects of applying adhesion prevention gel on the range of motion and pain after TKA. The Knee 2011; 18(2):104-107.
10. Seiichi O, Nishiyama T, Sakoda M et al. Development of carboxymethyl cellulose nonwoven sheet as a novel hemostatic agent. J Biosci Bioeng 2015; 119(6):718-723.

11. Yang Y, Liu X, Li Y, et al. A postoperative anti-adhesion barrier based on photoinduced imine-crosslinking hydrogel with tissue-adhesive ability. Acta Biomater 2017; 62:199-209.

12. Lawhorn KW, Howell SM. Principles for using hamstring tendons for anterior cruciate ligament reconstruction. Clinics in Sports Medicine 2007; 26(4):567-585.

13. Riaz O, Nisar S, Phillips H, Siddiqui A. Quantifying the problem of kneeling after a two incision bone tendon bone arthroscopic anterior cruciate ligament reconstruction. Muscles, Ligaments and Tendons 2015; 5(3)181-186.

14. Kjaergaad J, Faune LZ, Faune P. Sensibility loss after ACL reconstruction with hamstring graft. Int J Sports Med 2008; 29(6):507-511.

15. Charalambous CP, Kwaees TA. Anatomical considerations in hamstring tendon harvesting for anterior cruciate ligament reconstruction. Muscles, Ligaments and Tendons Journal $2012 ; 2(4): 253-257$ 